\title{
A Study on Boundary Detection Method for Separation of Coastal Seahorse Community Obtained by Drones
}

\author{
Kwang-Seong Shin ${ }^{1}$ and Seong-Yoon Shin ${ }^{2 *}$ \\ ${ }^{1}$ Dept. of Digital Contents Engineering, Wonkwang University, \\ 54383, Iksan, Korea \\ ${ }^{2 *}$ School of Computer Information \& Communication Engineering, Kunsan \\ National University, 54150, Kunsan, Korea \\ waver0920@wku.ac.kr,s3397220@kunsan.ac.kr
}

\begin{abstract}
Drones are becoming more commonly used for geological aerial photography.

This study used pixel-based image processing in order to distinguish seaweed colonies in a salt marsh on the west coast of South Korea after photographing them with a drone.

Also, in order to improve performance prior to detecting the edges, binarization is performed as a preprocessing step, and the edge detection result varies depending on how the threshold value is determined,

Generally, differentiation-based methods are widely used for edge detection and convolution mask methods for edge detection proposed by Sobel, Prewitt, Roberts, etc. for effectively implementing the concept of differentiation.

To clearly separate specific colonies in a video of west coast mudflat recorded by using a drone, this study compared the relationship between threshold values decided during the binarization process and convolution mask techniques for edge detection.
\end{abstract}

Keywords: Image binarization, boundary detection

\section{Introduction}

Recently, numerous geological and topographic image data are being produced by using aerial photography with drones. It is necessary to automatically separate certain areas and determine their size from the image.

In order to classify and classify regions in the target image, we use pixel-based processing of images, morphological processing of images, and geometric processing.

In this case, depending on which method is used, there are many differences in the perception of the person and the result. Maybe it's a different area than a person's cognition.

The field of pattern recognition, which belongs to the field of cognitive science and artificial intelligence, has been actively studied recently by expanding the field of deep learning based on Big Data.

However, learning requires a large amount of data to be accumulated.

This study used, among various image processing techniques, the pixel-based image processing technique in order to separate a certain area from the obtained image.

Pixel-based image processing converts a pixel value solely relying on the current pixel value, regardless of other pixel values.

Pixel-based processing is the most basic operation used in various fields such as image conversion, image enhancement, and compositing.

In this study, to separate a certain seaweed colony in an aerial photography of a salt marsh on the west coast of South Korea taken by a drone, edge detection was performed

Received (May 6, 2018), Review Result (July 8, 2018), Accepted (July 20, 2018) 
by using image processing and, subsequently, to determine the size of the detected area, a closed curve was detected by using an image contour algorithm.

Here, as a preprocessing step, grayscale conversion of the color image and binarization of the grayscale image are performed.

The image is binarized in order to remove noise and facilitate analysis of the image. The threshold value determined during binarization and selection and combination of edge detection algorithms determine how the seaweed colonies are finally separated.

In doing so, to prevent unnecessary edge detection, blurring and other methods are used to remove noise and histogram smoothing and various preprocessing operations are performed to improve the result. In other words, there is a substantial correlation between the preprocessing step and edge detection result.

High frequency and low frequency filtering methods are used to remove noise from the image.

The problem of the spatial domain can be considered as a frequency domain problem. The frequency in the frequency domain represents the degree of change in brightness or chromaticity of the image in the spatial domain. For example, a high frequency means that there are many changes in brightness or chromaticity, and in the frequency domain, the low frequency and high frequency components can be easily separated and the constant frequency region can be manipulated to affect the improvement of the original image. For example, removing the high frequency component smooths the image and removes the low frequency component, resulting in an image with a border highlighted.

In this study, effective boundary detection method was investigated to distinguish seahorse communities of the West Sea salt marshes photographed by drones. Boundaries were emphasized by eliminating low frequency components before binarization.

In this study, binarizations were performed by, once, using a threshold value determined based on Otsu's Method and, then, using a threshold value determined manually, respectively, prior to detecting the edge by using the convolution mask methods for edge detection proposed by Sobel, Prewitt, and Roberts.

Also, for performance evaluation, this study measured the effect of threshold value determined during the binarization process by the number of closed curves created by using the image contour algorithm, on the edge detection result

\section{Related Works}

Image processing techniques are often used to identify and search objects through image processing. Various preprocessing processes are performed to improve image processing performance.

Sarker used an adaptive Gaussian mixture model for background subtraction in a complex environment [14], and Seok-Wun used a color histogram to search for images. And the sensitivity of the acquisition process [1].

In order to improve the edge detection performance in complex images, Choi Hyun et al. Have compared the noise removal by high frequency filtering and low frequency filtering by distorting the road boundary detection in high resolution satellite images and removed specific frequency components to improve boundary detection performance in the preprocessing step[2].

It is possible to perform processing such as blurring or sharpening an image by filtering a specific frequency after converting the image into a frequency domain. There are various filters, but a low-pass filter that passes only low-frequency components and removes high- A high-pass filter for communicating a highfrequency component, a band-pass filter for passing only a specific band, and a band-stop filter for removing a specific band. The low-pass filter makes the image blurred and the high-pass filter sharpens the image. 
In an environment where noise is present, maximizing image boundaries and detail is one of the most important factors in eliminating noise. Yoo, Jong-Sang et al. Have attempted to remove image noise using a bilateral filter to remove noise from the original image, and this method proved to be a method of preserving the boundaries and detail of the image. Due to this characteristic of the bilateral filter, research on the elimination of the image noise through the bilateral filter is currently underway. In order to improve the ability to highlight the advantages of the bilateral filter, which is the advantage of bilateral filters, they proposed a more effective bilateral filter image noise reduction method using the simplest known image boundary detection algorithm[3].

\subsection{Binarization}

A binarized image is an image that expresses all pixels only in black and white.

Briefly, if the gray scale is represented by expressing only 256 levels of brightness, the binarized image is an image that expresses brightness only with 0 and 1 , that is, full black and white.

The binarization of an image refers to a process of making a pixel having a low value as a black (0) and a pixel having a high value as a back (255) based on a certain threshold value.

The binarized image is used to detect the characteristics of the objects contained in the image and is known as a basic work for application in license plate recognition, document recognition, and the like. Using a binarized image, you can clearly show the character part of the scanned book image. Also, the file size of the binary image becomes smaller.

Binarization is a two-class classification process in which the subjects are classified into either 0 or 1 .

It is performed as a preprocessing step for edge detection and, in general, based on Otsu's Method, the threshold value $\mathrm{T}$ is automatically determined as either 0 or 1.

To determine the threshold value $\mathrm{T}$, the binarization method looks for $\mathrm{T}$ that either minimizes the intra-class variance between two classes or maximizes the inter-class variance when dividing image pixels into two classes.

First, binarization is performed to detect edges in an 8-bit grayscale image, and the result varies depending on the threshold value, as all pixels that are brighter than the threshold value are converted into 1 and those darker than the threshold value into 0 . Determination of the threshold value in this process is critical. Therefore, in general, Otsu's Method is commonly used to automatically set the threshold value based on brightness distribution of the image [4].

$\mathrm{Sa}$ et al., believed the traditional Otsu's Method does not show adequate performance when dividing a target in the background during the strip detection process in calculating the threshold value and, therefore, proposed an improved threshold segmentation method based on the maximum variance algorithm [5].

Ranjani et al., proposed a hybrid approach for document image binarization, which applies the L0 gradient minimization algorithm and smooths image in order to prevent quality degradation of the background image while the foreground is improved by using local contrast [6].

Zayed et al., proposed an effective document image binarization method to overcome various quality degradation factors such as bleed-through, aging, and poor lighting conditions [7].

Bae, Jong-Wook, et al., Proposed a method to recognize the amount and number of coins in order to implement the coin calculation system. In order to increase the recognition rate of the coins, binarization was performed and the size information of the coins was used. Additionally, the histogram information of the image was used to increase the recognition rate [8]. 


\subsection{Edge Detection}

Edge detection is one of the main steps in image processing, image recognition, and computer vision as well as in image analysis, and can be used for a variety of fields including face recognition, industrial technology, system search, and military system.

A contour is a boundary line having a different intensity from the viewpoint of image processing, and refers to a case where the brightness of a pixel changes more than a threshold value. The amount of change in brightness is used to find outlines in the image.

The magnitude of the slope can be used in the first derivative and the brightness and darkness in the second derivative can be judged as the sign of the slope. In the image, we use a mask for differential calculations, which is a kind of filter with the same effect as the differential operator.

In most computer vision systems, the importance of effective and reliable edge detection methods is growing day by day[9].

This type of algorithm is composed of a series of mathematical methods that aim to identify points at which the brightness changes rapidly or discontinuous movement is detected in the presented image.

In other words, in images, edges refer to a boundary between areas that have different gray levels and are mainly found between an object and background or an object and another object [10].

Conventional edge detection methods include Sorbel's and Prewitt's methods, which apply vertical or horizontal masks, and Robert's, which applies masks in different diagonal directions [11-13].

Roberts's mask is smaller than other masks; therefore, it performs more efficient operations but is more sensitive to noise. Prewitt's mask was designed to be more sensitive to horizontal and vertical boundary lines than to diagonal lines. By contrast, Sobel's mask is more sensitive to diagonal boundary lines than to horizontal and vertical lines.

In Prewitt's mask, the value is either +1 or -1 , regardless of the pixel's distance from the center of the mask, while, in Sobel's mask, pixels that are closer to the mask center are either +2 or -2 and those farther from the center either +1 or -1 , thereby, allowing for more precise detection of the boundary lines. It is because, in convolution operation, pixels that are closer to the center pixel are more influential.

The main disadvantage of this type of edge detectors is that they are sensitive to noise and inaccurate[14].

Although these methods use simple operators and are able to easily detect edges in an area with a considerable gradient change, the processing results are less than satisfactory because the same mask is applied to the entire image regardless of the pixel distribution, etc., [15-18].

Unlike the boundary detection method using the differential operator to perform boundary detection in an image, Kim et al. Refer to two neighboring pixel information in a binarized image, compare the four neighboring pixel patterns with predefined boundary pixel patterns, they propose a method to detect the boundaries of the binarized image by detecting the boundaries of the detected search pixels and detecting the non - visited pixels to detect the boundaries of the next region.

In case of performing boundary detection using differential operator when performing image recognition, it is possible to omit the thinning process which is the next step of boundary detection, and the boundary detection time can be shortened compared to the existing boundary boundary tracking method [19].

Kim et al., propose a new algorithm for the classification of binary classification problems, such as the Convolutional Neural Network (CNN), Recurrent Neural Network (RNN) and Deep Boltzmann Machine (DBM) Function selection, and the 
relationship between various network design issues. We also analyzed the effects of Multi-Layer Perceptron, which is a traditional artificial neural network, CNN, which is a deep learning algorithm, and Long Short-Term Memory, which is a variation of RNN. The performance of the proposed method is verified by comparing the performance with the binary classification problem[20].

\section{Test and Results}

The test in this study was performed by using MATLAB2015a and Visual C++ Open CV.
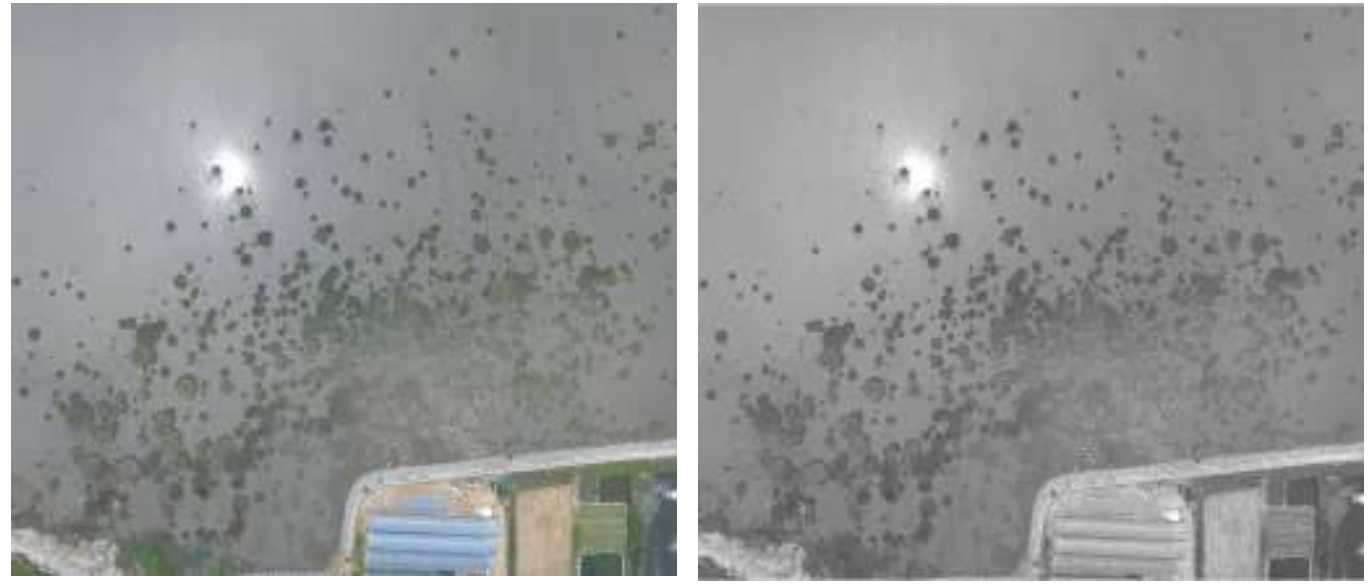

Figure 1. Source Image(Left) and Grayscale Image(Right)

By using MATLAB, the original color image was converted into grayscale prior to performing binarization in which the threshold value $\mathrm{T}$ was automatically determined based on Otsu's Method and binarization based on the threshold values of 110, 130, and 140.

For the binarized images based on each threshold value, edge detection was performed by using the Sobel, Prewitt, and Roberts convolution masks.

Due to the strong contrast with the sunlight reflected on the mudflat in the original image, in the result created by using automatic binarization based on Otsu's Method, it was not possible to separate the seaweed colony with the naked eye.

When the threshold value was set at 110,130, and 140, the results, while varying, enabled easy edge detection.

For the results that were binarized based on different threshold values, edge detection was performed by using the three different convolution masks.

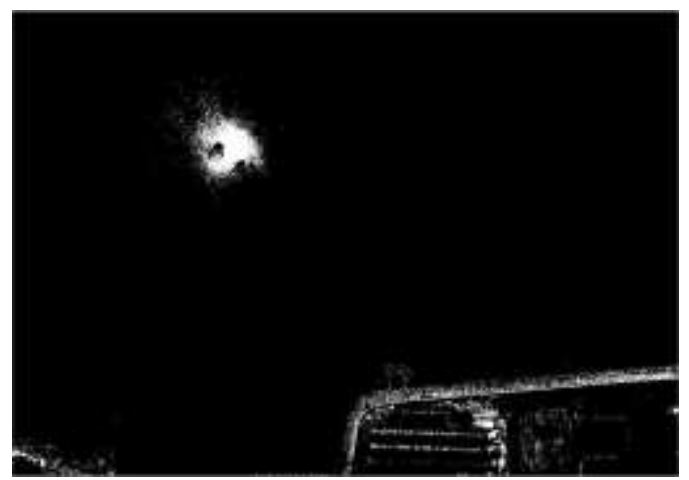

Otsu Method

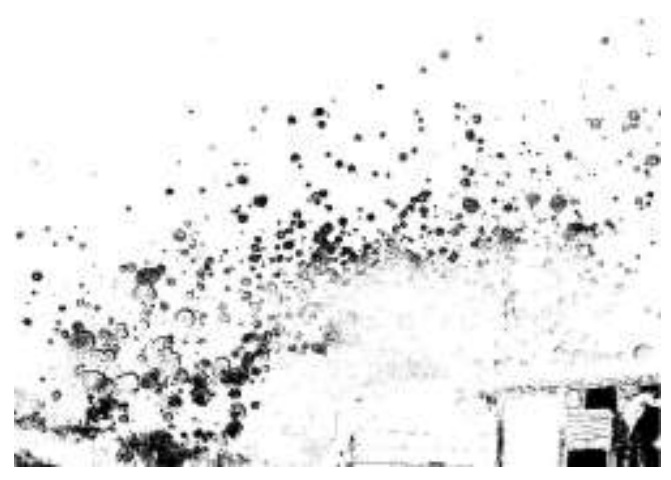

$\mathrm{T}=130$ 


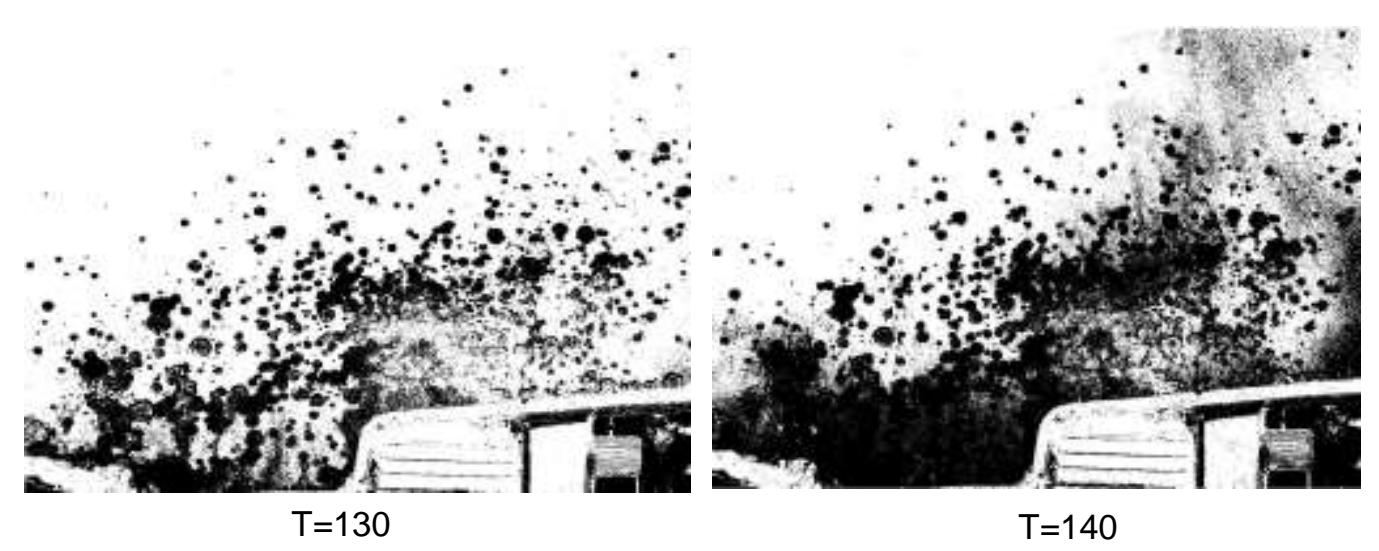

Figure 2. Binariztion

When thresholds were determined automatically by the Otsu method, prewitt, Roberts, and Sobel operations were performed for each mask. When the threshold is 110, 130, and 140 , boundary detection is performed for each mask.

When the three convolution masks were applied to the original image, there was no difference in edge detection performance.

The performance was evaluated based on the number of closed curves created by the image contour algorithm.

Image contour refers to connecting the outer edge of an area that has the same color or same color intensity.

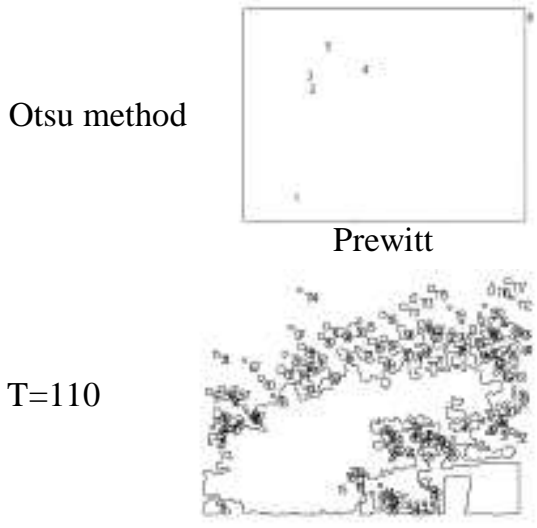

Prewitt

$\mathrm{T}=130$

$\mathrm{T}=140$

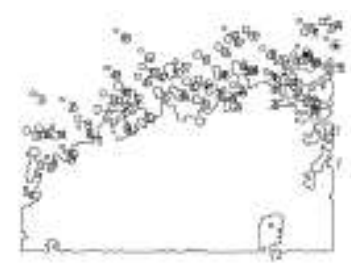

Prewitt

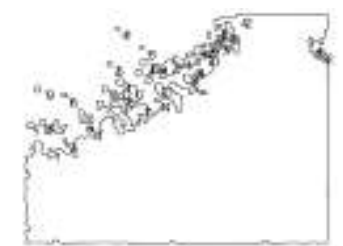

Prewitt

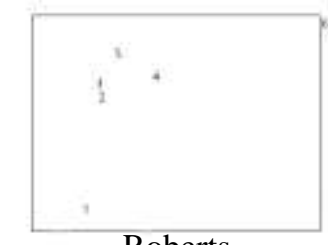

Roberts

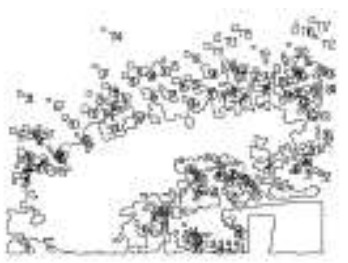

Roberts

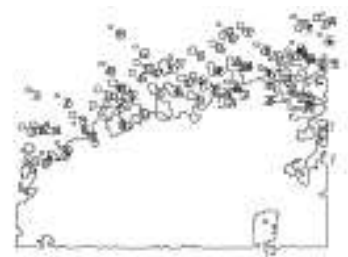

Roberts

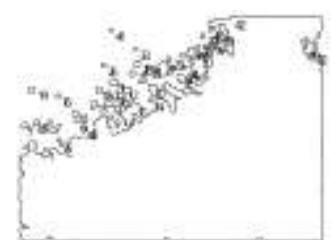

Roberts
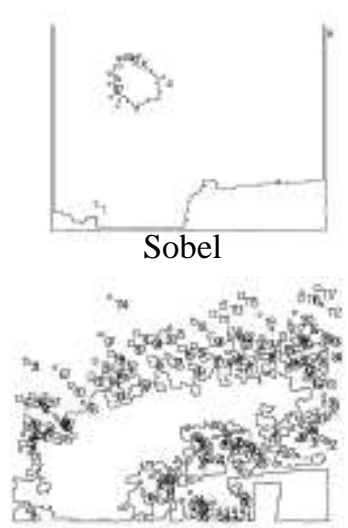

Sobel

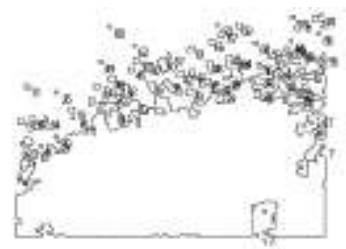

Sobel

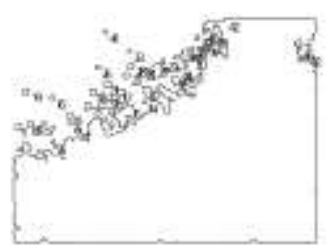

Sobel

Figure 3. Results of Closed Curve by Edge Detection Mask 
It is useful for analyzing shape of an object or recognizing an object in an image.

In this study, the function cv2.findContours() in OpenCV, which uses the Suzuki85 algorithm, was used.

It is shown that binarization by automatic determination of threshold value by Otsu method is not always the best.

And 110, 130, and 140 as representative values in which the distinction becomes clear while changing the threshold value.

The closed curve detection results based on binarization of Otsu's Method and on threshold values 110,130 , and 140 are as follows:

Although there was no significant difference in edge detection results among the three different convolution masks, considerable difference was observed according to the threshold value in binarization performed as a preprocessing step.

\section{Conclusions}

Using pixel-based image processing to separate a certain area in an image involves different variables. The results can vary widely from human detection, because the method does not recognize pattern.

Various factors need to be taken into account in order to easily separate a particular area with the aid of computer.

This study demonstrated a close relationship between edge detection performance and preprocessing of the input image when using convolution masks, as which are a type of of pixel-based operation that is commonly used for separating a certain area in an image.

In this study, edge detection performance according to different threshold values determined during binarization was compared by using the image contour algorithm.

The result of this study showed that, when performing binarization, Otsu's Method does not always provide an optimal condition for edge detection and that, in binarization of images with strong contrast, the relationship between the set threshold value and edge detection result is especially sensitive.

Pixel - based computation for morphological image processing has advantages such as fast computation and low capacity, but it has a limitation that it does not reach human 's cognitive level.

In future research, we intend to approach the perception level of human through deep data based deep learning method as a method to detect and recognize a specific pattern in order to distinguish the community from the geological image taken by the drone.

\section{Acknowledgments}

This paper is a revised and expanded version of a paper entitled "Grain size analysis using image processing" presented at AWITC 2018, February 9, 2018 Tower Hill Hotel, Busan, Korea.

\section{References}

[1] S.-W. Ha, "A New Method for Color Feature Representation of Color Image in Content-Based Image Retrieval - 2D Projection Maps", Journal of information and communication convergence engineering, vol. 2, no. 2, (2014), pp. 123-127.

[2] H. Choi and I.-J. Kang, "Comparative Analysis of LPF and HPF for Roads Edge Detection from High Resolution Satellite Imagery", Journal of Korean Society for Geospatial Information System, vol. 14, no. 3, (2006), pp. 3-11.

[3] J. S. Yoo, S. Lee, G. Jeon and J. Jeong, “Adaptive Bilateral Filtering for Image Denoising using Edge Detection", In Proceedings of the Korean Society of Broadcast Engineers Conference, The Korean Institute of Broadcast and Media Engineers, (2013), pp. 270-273.

[4] N. Otsu, "A Threshold Selection Method from Gray-Level Histograms", IEEE Transactions on Systems, Man, and Cybernetics IEEE Trans. Syst., Man, Cybern. Systems, Man and Cybernetics, IEEE Transactions, vol. 9, no. 1, (1979), pp. 62-66. 
[5] J. Sa, X. Sun, T. Zhang, H. Li and H. Zeng, "Improved Otsu segmentation based on sobel operator", 2016 3rd International Conference on Systems and Informatics (ICSAI) Systems and Informatics (ICSAI), 2016 3rd International Conference, (2016), pp. 886-890.

[6] J. Ranjani, "Bi-level thresholding for binarisation of handwritten and printed documents", IET Computer Vision, vol. 9, no. 1, (2015), pp. 41-50.

[7] M. Zayed, A. Ouari, M. Derraschouk and Y. Chibani, "An effective hybrid thresholding technique for degraded documents images binarization", 2011 International Conference for Internet Technology and Secured Transactions Internet Technology and Secured Transactions (ICITST), 2011 International Conference, (2011), pp. 460-465.

[8] J.-W. Bae and S.-H. Jung, "Coin Calculation System Using Binarization and Hue Histogram", KIISE Transactions on Computing Practices, vol. 21, no. 6, (2015), pp. 424-429.

[9] R. Nevatia, "A color edge detector and its use in scene segmentation", IEEE Trans. Syst., Man, Cybern., vol. SMC-7, no. 11, (1977), pp. 820-826.

[10] C.-Y. Lee and N.-H. Kim, "Study on Edge Detection Method using Modified Directional Masks", Journal of the Korea Institute of Information and Communication Engineering, vol. 18, no. 11, (2014), pp. 2779-2785.

[11] L. Mero and Z. Vassy, "A Simplified and Fast Version of the Hueckel Operator for Finding Optimal Edges in Pictures", Proceeding of International Joint Conferences on Artificial Intelligence, Tbilisi Georgia: USSR, (1975), pp. 650-655.

[12] P. Meer and B. Georgescu, "Edge Detection with Embedded Confidence", IEEE Transactions on Pattern Analysis and Machine Intelligence, vol. 23, no. 12, (2001), pp. 1351-1365.

[13] B. Kurt and M. Gokmen, "Goal Oriented Edge Detection", 23rd International Symposium on Computer and Information Sciences, Istanbul:Turkey, (2008), pp. 1-5.

[14] B. Popa, "Study about the edge detection algorithm and its applications", 2017 18th International Carpathian Control Conference (ICCC) Carpathian Control Conference (ICCC), 2017 18th International, (2017), pp. 417-422.

[15] B. Kaur and A. Garg, "Mathematical Morphological Edge Detection for Remote Sensing Images", 3rd International Conference on Electronics Computer Technology, Kanyakumari:India, vol. 5, (2011), pp. 324-327.

[16] R. C. Gonzalez and R. E. Woods, "Digital Image Processing", 3rd ed. Pearson Education, Inc., (2008).

[17] R. C. Gonzalez, R. E. Woods and S. L. Eddins, "Digital Image Processing Using MATLAB", 2nd Prentice-Hall, Inc., (2004).

[18] Md. M. Kamal Sarker, C. Weihua and M. Kyou Song, "Detection and Recognition of Illegally Parked Vehicles Based on an Adaptive Gaussian Mixture Model and a Seed Fill Algorithm”, Journal of information and communication convergence engineering, vol. 13, no. 3, (2015), pp. 197-204.

[19] S.-H. Kim, S.-B. Nam and H.-J. Lim, "An improved area edge detection for real-time image processing", Journal of the Korea Society of Computer and Information, vol. 14, no. 1, (2009), pp. 99-106.

[20] K. Kim, B. Lee and J. W. Kim, "Feasibility of Deep Learning Algorithms for Binary Classification Problems", Journal of intelligence and information systems, vol. 23, no. 1, (2017), pp. 95-108.

\section{Authors}

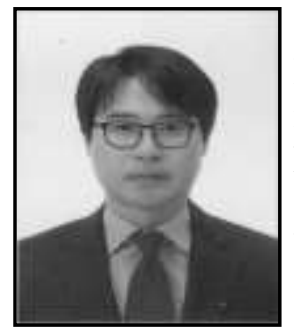

Kwang-Seong Shin, received his master's degree from the Dept. of Computer Engineering of Chonbuk National University in 2005 and his Ph.D. from the Dept. of Computer Information Engineering of Kunsan National University in 2014.

Currently, he is an associate professor at Wonkwang University. His research interests are Image Processing, Computer Vision, AR, VR and MR.

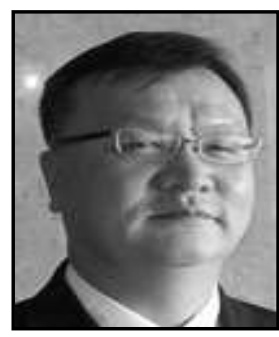

Seong-Yoon Shin, received his M.S. and Ph.D degrees from the Dept. of Computer Information Engineering of Kunsan National University, Kunsan, Korea, in 1997 and 2003, respectively. From 2006 to the present, he has been a professor in the School of Computer Information \& Communication Engiineering. His research interests include Image Processing, Computer Vision, and Multimedia. 